\title{
Equilibria on a circular market when consumers do not always buy from the closest firm
}

\author{
Dominic Keehan ${ }^{1}$ - Dodge Cahan ${ }^{2}$. John McCabe-Dansted ${ }^{3}$. \\ Arkadii Slinko ${ }^{1}$ (D)
}

Received: 21 February 2021 / Accepted: 22 January 2022 / Published online: 21 February 2022

(c) The Author(s) 2022

\begin{abstract}
We study spatial competition by firms which is often studied in the context of linear markets where customers always shop at the nearest firm. Here, customer behavior is determined by a probability vector $\mathbf{p}=\left(p_{1}, \ldots, p_{n}\right)$ where $p_{i}$ is the probability that a customer visits the $i$ th closest firm. At the same time, the market is circular a là Salop (Bell J Econ 10(1):141-156, 1979), which has the advantage of isolating the impact of customer shopping behavior from market boundary effects. We show that non-convergent Nash equilibria, in which firms cluster at distinct positions on the market, always exist for convex probability vectors as well as probability vectors exhibiting a certain symmetry. For concave probability vectors, on the other hand, we show that non-convergent Nash equilibria are unlikely to exist.
\end{abstract}

Keywords Salop circular city model $\cdot$ Competition of firms $\cdot$ Non-convergent Nash equilibria

Dodge Cahan is currently employed by Amazon.com Inc. His contribution was prior to joining Amazon and does not represent Amazon's views.

$凶$ Arkadii Slinko

a.slinko@auckland.ac.nz

Dominic Keehan

dkee331@aucklanduni.ac.nz

Dodge Cahan

cahan@ualberta.ca

John McCabe-Dansted

gmatht@gmail.com

1 Department of Mathematics, University of Auckland, Auckland, New Zealand

2 Department of Economics, University of Alberta, Edmonton, Canada

3 School of Computer Science and Software Engineering, University of Western Australia, Perth, Australia 
JEL Classification C72 · D72

\section{Introduction}

Hotelling's (1982) classic model of spatial competition by firms on a linear market has been a cornerstone of economic theory, giving rise to countless extensions and generalizations. In many markets, a linear market is natural-e.g., the so-called Main Street model (Downs 1957; Eaton and Lipsey 1975; Graitson 1982). But markets are more varied in nature and the topology of the space where the competition takes place can vary. One such alternative space used in the classical circular city model, first introduced by Salop (1979), provides a natural representation for a wide variety of situations (such as a beltway around a city center) and has been a workhorse in the spatial competition literature ever since. The circular market has also been of interest because it allows the study of various market forces in isolation of boundary effects, which can be substantial (Aoyagi and Okabe 1993).

Salop's circular city model assumes that a customer always buys from the nearest firm. This assumption leads to the fact - exactly as in the Hotelling-Downs modelthat no more than two firms can occupy the same location (Peeters et al. 2010, 2016). This contradicts the observable behaviour of firms that often cluster in larger numbers (e.g., fast food restaurants). To get a more realistic set of Nash equilibria we must relax this assumption and allow customers to patronise firms other than the closest one with some probability.

This approach originated with Cox (1987) in a political candidate positioning framework, where he studied political competition under a class of electoral systems known as scoring rules. Though Cox's setting was very different, as Myerson (1999) wrote, "there are logical similarities between political competition and market competition, and so analytical skills that have been sharpened by the study of either arena may be applied to offer new insights in the other arena." In the economics setting, indeed, a scoring rule can be interpreted as a probability vector describing how likely customers are to shop at more distant firms. The application of this assumption to the firm interpretation has been explored primarily in the case of a linear market (Cahan and Slinko 2017, 2018; Cahan et al. 2018).

To justify the implementation of Cox's idea in the case of markets we need to look at the micro-foundation of Salop's model which is rooted in the distances between the consumer and the firms. The consumer's utility of purchasing from firm $i$ is $v-c d_{i}$, where $v$ is the value of the object, $d_{i}$ is the distance between the consumer and firm $i$ and $c$ is the cost $c$ of travel per unit of distance. Since all firms are selling identical objects, the firm which is closest to the consumer offers him the highest utility, which explains why he purchases from that firm with probability 1. Implementing Cox's idea does not change the micro-foundation, it only introduces an element of randomness to the model. Indeed, in our model the consumer's utility of purchasing from firm $i$ is $v-c\left(d_{i}+\epsilon_{i}\right)$, where $\epsilon_{i}$ is a random value. This random value may be the result of a number of external factors such as weather, traffic conditions or available transport. For example, a rush hour traffic jam will delay the consumer and is equivalent to travelling a longer distance. As a result, the vector of probabilities $(1,0, \ldots, 0)$ in 
Salop's model becomes $\left(p_{1}, \ldots, p_{n}\right)$, where $p_{i}$ is the probability the consumer buys from the $i$ th distant firm. But the overall logic remains the same: the shortest distance is a paramount concern.

The main pattern emerging from the literature is that "best-rewarding" voting rules 1 that, roughly speaking, reward top ranked candidates while treating low ranked candidates similarly, encourage candidates to adopt differentiated policy platforms. On the other hand, "worst-punishing" voting rules, which treat top ranked candidates similarly while punishing poorly ranked candidates, encourage candidates to adopt similar policy positions. In the firm interpretation, this amounts to saying that: when the probability that a customer shops at more distant firms drops off quickly in the firm's ranking, then firms locate at diverse locations; on the other hand, when there is a slow drop off in probability of patronage as firms become more distant, then firms are encouraged to agglomerate at a single location (e.g., a mall or city center). This result can be made quantitatively precise by defining Cox's threshold of diversity which we call the Cox value or $c$-value. We assume that the society of customers is characterised by a vector of probabilities $\mathbf{p}=\left(p_{1}, \ldots, p_{n}\right)$, such that a customer buys from the $i$ th most distant retailer with probability $p_{i}$. We assume $p_{1} \geq p_{2} \geq \cdots \geq p_{n}$ and $p_{1}>p_{n}$. The Cox value is defined as $c(\mathbf{p}, n)=\frac{p_{1}-\bar{p}}{p_{1}-p_{n}}$ so that best-rewarding vectors have $c(\mathbf{p}, n)$ greater than one half, worst-punishing vectors have $c(\mathbf{p}, n)$ less than one half, and intermediate vectors have $c(\mathbf{p}, n)$ equal to one half. Intuitively, having bestrewarding vector $\mathbf{p}$ means that the market share of a particular firm is affected more by a relocation of its neighboring firms than more distant firms.

On a linear market, there are broad classes of probability vectors for which equilibria do not exist, while for others agglomerative, non-convergent equilbria can be constructed (Cahan and Slinko 2017, 2018; Cahan et al. 2018). Results are often influenced, however, by the shape of the market and its boundaries (Eaton and Lipsey 1975; Aoyagi and Okabe 1993). Without the "peripheral firm advantage" of the linear model, our expectation is that equilibria in the circular model will be more numerous and exist for larger families of probability vectors.

The goal of this paper, then, is to investigate under which probability vectors equilibria on a circular market exist and whether they are convergent or not. We show that for convex probability vectors-which are best-rewarding — non-convergent Nash equilibria always exist and are similar to the classical case where $\mathbf{p}=(1,0, \ldots, 0)$. For intermediate vectors, we describe a class of symmetric probability vectors for which every profile is a Nash equilibrium. For the class of concave probability vectorswhich are worst-punishing-we show the existence of convergent Nash equilibria and hypothesise that there are no non-convergent ones. Hinting at the validity of our hypothesis, we prove that under concave probability vectors we can rule out the possibility of bipositional Nash equilibria.

\footnotetext{
1 This terminology is due to Myerson (1999).

2 The classical model then corresponds to the probability vector $(1,0, \ldots, 0)$.
} 


\section{The model}

There are $n$ firms that must choose their locations on the circular market, modelled as a circle of circumference of length 1 with a unit mass of consumers distributed uniformly along it. Firms produce a homogeneous good with a fixed mill price and consumers have inelastic demand-everyone demands one unit. However, consumers do not always buy from the nearest firm. With probability $p_{i}$, a consumer buys from the $i$-th nearest firm. This could be because consumers sometimes travel for unrelated reasons and happen to find it more convenient to visit another firm. Or, the product might sometimes simply be out of stock at a given store. Again, this is formalised through a probability vector $\mathbf{p}=\left(p_{1}, \ldots, p_{n}\right)$, where $p_{i} \geq p_{i+1}$ with $p_{1}>p_{n}$, and $\sum_{i} p_{i}=1 .^{3}$ Thus, rather than incorporating a random utility shock as is done in the probabilistic literature (De Palma et al. 1990), we use a deterministic function that captures a similar notion. The probability implied is of an ordinal nature-there is no dependency on absolute distance, as occurs in most of the literature on probabilistic voting (see, e.g., Coughlin 1992; Duggan 2005).

It has to be noted that the absence of dependency on absolute distance is the salient feature of models of competition of firms in the strand of literature to which this paper belongs (see, e.g., Eaton and Lipsey (1975), Salop (1979)). Indeed, in the paper of Salop (1979), which is the closest to us, a consumer always buys from the closest retailer, and, if another retailer is only marginally further, our consumer never buys from that firm. In contrast, in all models of probability voting both firms will be patronised with almost equal probability.

In equilibrium, all firms can occupy the same location (a convergent Nash equilibrium or CNE) or not (a non-convergent Nash equilibrium or NCNE). NCNE often appear clustered. For example, fast food restaurants and gas stations of various chains are usually located in clusters that are spread intermittently along a main street. This effect was called the 'Principle of Local Clustering' by Eaton and Lipsey (1975). However, when consumers always go to the nearest firm, such equilibrium can exist only if the number of firms at each location is one or two (Eaton and Lipsey 1975; Graitson 1982). By incorporating the possibility that consumers purchase at firms other than the nearest one, we show that equilibria with multiple firms at the same location can arise in a very simple way in a broad class of cases which makes our model a better fit for the observed behaviour of firms.

In the political competition setting, a vector $\mathbf{s}=\left(s_{1}, \ldots, s_{n}\right)$ of scores represents a points-based electoral system where voters rank all the candidates and $s_{i}$ points are assigned to the voter's $i$ th ranked candidate. Though we focus on the firm interpretation, by analogy we sometimes use the following terminology: $\mathbf{p}=(1,0, \ldots, 0,0)$ is the plurality probability vector; $\mathbf{p}=\frac{1}{k}(1, \ldots, 1,0, \ldots, 0)$ is $k$-approval; $\mathbf{p}=$ $\frac{2}{(n-1) n}(n-1, n-2, \ldots, 1,0)$ is Borda; and, $\mathbf{p}=\frac{1}{A}((n-1) a+b, \ldots, a+b, b)$, where $A=\frac{n(n-1)}{2} a+b$, is a linear probability vector. ${ }^{4}$

\footnotetext{
3 Of course, every consumer would have their own vector of probabilities but we assume that we have managed to aggregate individual vectors into a single societal vector of probabilities.

4 Borda is a special case of a linear probability vector with $(a, b)=(0,1)$.
} 
In this paper we focus on two broad classes of probability vectors representing two plausible descriptions of consumer behavior: (i) a convex probability vector $\mathbf{p}$, where $p_{i-1}-p_{i} \geq p_{i}-p_{i+1}$ for all $i$; and, (ii) an intermediate probability vector $\mathbf{p}$, where $p_{i-1}-p_{i}=p_{i}-p_{i+1}$ for all $i$. As an example, it would be quite natural to assume that the probability of a customer patronising the $i$ th distant firm falls exponentially with $i$, i.e., $p_{i}=p^{-\lambda_{i}}$ for $\lambda_{1}<\lambda_{2}<\ldots$, which defines a strictly convex probability vector. The case of a concave probability vector $\mathbf{p}$, where $p_{i-1}-p_{i} \leq p_{i}-p_{i+1}$ for all $i$, is also considered but we argue that it is less realistic. For this case we have only partial results, the main of which is to rule out any possibility of bipositional Nash equilibria.

For the rest of this paper $n$ will mean the total number of firms, $m$ is the total number of occupied locations, and $n_{i}$ is the total number of firms in the $i$ th location. Let us first define the market of a location occupied by at least one firm. This is the expected measure of customers for which this location is the nearest of all occupied locationsthus, customers will buy from one of the firms at this location as their top preference. If the firm is not alone at this location, then customers coming to this location will buy with equal probability from any firm at that location. Hence the market of a firm is the market share of its location divided by the number of firms at that location (we must be careful not to mix these two things).

Definition 1 A strategy profile $x=\left(\left(x_{1}, n_{1}\right), \ldots,\left(x_{m}, n_{m}\right)\right)$ of firms characterises the locations $x_{1}, \ldots, x_{m}$ occupied by firms and indicates the multiplicities $n_{1}, \ldots, n_{m}$, i.e., the number of firms at each location.

Definition 2 A strategy profile is a Nash Equilibrium (NE) if no one firm can increase its market share by moving to another location. The NE is convergent (CNE) if all firms are at the same location; if the firms occupy more than one location, the NE is called non-convergent (NCNE). If a strategy profile $x=\left(\left(x_{1}, n_{1}\right), \ldots,\left(x_{m}, n_{m}\right)\right)$ is a Nash equilibria, then we say that it is of type $\left(n_{1}, \ldots, n_{m}\right)$.

\section{A brief survey}

In this section we give a short, more specific account of what is known about the linear and circular market cases. In this classical Hotelling setting, each firm only receives customers from its 'market', i.e., the set of consumers for which that firm is the closest or tied for closest. If $k$ firms are co-located, their market would be $k$ times smaller than if it were a single firm as they share consumers equally.

In the case of a linear, unit interval market, NCNE are not so easy to come by, but have been fully characterised (Eaton and Lipsey 1975; Denzau et al. 1985). When we consider probability vectors beyond plurality, Cahan and Slinko (2017) characterise equilibria for $n \leq 5$ and also investigate a range of classes of probability vectors, some of which permit NCNE.

There are obvious similarities and dissimilarities between the circular market and the linear bounded market. In general, the circular case appears to be easier to deal with due to the lack of boundary conditions. Eaton and Lipsey (1975) (see also Graitson 
Fig. 1 Left and right half-markets $L(x)$ and $R(x)$ at location $x$

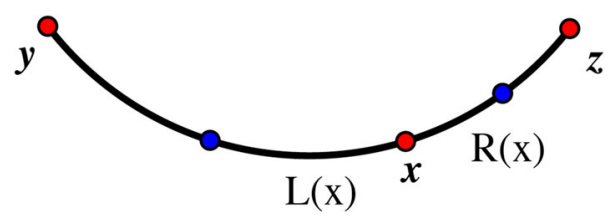

1982) studied this problem for the plurality probability vector. Their results for the circular market can be summarised as follows:

(i) There exist an infinite number of equilibria, whatever the number of firms may be;

(ii) In equilibrium, no more than two firms can be co-located;

(iii) In equilibrium, all firms may be isolated (e.g., in a fully symmetric arrangement).

Let us consider any three neighboring occupied locations $x, y, z$ on the circle as shown in Fig. 1.

The full market $L(x) \cup R(x)$ of location $x$ consists of half of the arc $y x$ which is called left half-market of $x$ and half of the arc $x z$ which is called right half-market of $x$. If $x$ and $y$ are neighbouring occupied locations (there are no other firms if we travel from $x$ to $y$ counterclockwise), then $R(x)=L(y)$.

Peeters et al. (2010) gave necessary and sufficient conditions for a strategy profile of firms to be in equilibrium (Theorem 5 of that paper). For completeness of exposition we will prove this theorem.

Theorem 1 A strategy profile $x=\left(\left(x_{1}, n_{1}\right), \ldots,\left(x_{m}, n_{m}\right)\right)$ is a NCNE iff for all $i \in\{1, \ldots, m\}$ we have $n_{i} \in\{1,2\}$ and the following two conditions are satisfied:

(I) If $n_{i}=1$, then $L\left(x_{i}\right)+R\left(x_{i}\right) \geq \max \left(L\left(x_{j}\right), R\left(x_{j}\right)\right)$ for all $j \neq i$;

(II) If $n_{i}=2$, then $L\left(x_{i}\right)=R\left(x_{i}\right) \geq \max \left(L\left(x_{j}\right), R\left(x_{j}\right)\right)$ for all $j \neq i$;

Proof Suppose there are $k \geq 2$ firms at location $x$. Then any firm at this location has $\frac{1}{k}(L(x)+R(x))$ customers and suppose $L(x) \geq R(x)$. Then by moving incrementally to the left any firm at this location will get $L(x) \geq \frac{1}{2}(L(x)+R(x))>\frac{1}{k}(L(x)+R(x))$, if $k>2$. Thus, this deviation is profitable unless $k=2$ and $L(x)=R(x)$.

Assume now that $n_{i} \in\{1,2\}$. If a firm moves from location $x_{i}$ to the interval between neighboring occupied locations $x_{j}$ and $x_{j+1}$, then it gets $\frac{1}{2}\left(L\left(x_{j}\right)+R\left(x_{j+1}\right)\right)=$ $L\left(x_{j}\right)=R\left(x_{j+1}\right)$. Thus it can get an advantage if and only if either (I) or (II) does not hold. If the firm from location $x_{i}$ joins a firm at location $x_{j}$, then it would get at most $\frac{1}{2}\left(L\left(x_{j}\right)+R\left(x_{j}\right)\right)$ which does not exceed $\max \left(L\left(x_{j}\right), R\left(x_{j}\right)\right)$, i.e., it is profitable if and only if (I) and (II) do not hold.

The cases of three or four firms can be clarified.

Three firms There are two possible types of equilibria:

1. Two opposite locations are occupied, one with two firms and one with a single firm;

2. Three firms occupy three different locations each of which is not on an arc whose length is larger than half of the length of the circle. 
Four firms There are three possible types of equilibria:

1. Two opposite locations are occupied, each with paired firms;

2. Four firms occupy three different locations, i.e., $x=\left(\left(x_{1}, 1\right),\left(x_{2}, 2\right),\left(x_{3}, 1\right)\right)$ so that $\operatorname{arc} x_{1} x_{3} \leq \operatorname{arc} x_{1} x_{2}=\operatorname{arc} x_{2} x_{3}$;

3. Four firms occupy four different locations such that (I) and (II) are satisfied (multiple equilibria).

Peeters et al. (2010) show that for any number of firms on a circle there is a NCNE. Moreover it can be shown that for any tuple $\left(n_{1}, \ldots, n_{m}\right)$, where $n_{i} \in\{1,2\}$, there is a NCNE of the form $x=\left(\left(x_{1}, n_{1}\right), \ldots,\left(x_{m}, n_{m}\right)\right)$.

\section{Nash equilibria: initial considerations}

In the general case, the parameter $c(\mathbf{p}, n)=\frac{p_{1}-\bar{p}}{p_{1}-p_{n}}$, where $\bar{p}=\frac{1}{n}\left(p_{1}+\cdots+p_{n}\right)=\frac{1}{n}$, plays an important role in the existence of Nash equilibria and their properties (Cox 1987; Cahan and Slinko 2017, 2018; Cahan et al. 2018).

Note that for a fixed number $n$ of firms $\frac{1}{n} \leq c(\mathbf{p}, n) \leq \frac{n-1}{n}$. For plurality, Borda, $k$-approval and antiplurality (also known as negative voting or $(n-1)$-approval), the parameter $c(\mathbf{p}, n)$ takes values $\frac{n-1}{n}, \frac{1}{2}, \frac{n-k}{n}$ and $\frac{1}{n}$, respectively. Thus, plurality and antiplurality are the two extremes.

\subsection{Necessary and sufficient conditions for a CNE}

Cox showed that convergent Nash equilibria (CNE) for the linear bounded market exist if and only if $c(\mathbf{p}, n) \leq \frac{1}{2}$. That is, if and only if the vector is worst-punishing (Myerson 1999) or intermediate (in the case of equality). Exactly the same result is true for the circular market.

Theorem 2 For a circular market CNE exist if and only if $c(\mathbf{p}, n) \leq \frac{1}{2}$.

Proof If all $n$ firms are co-located, then all of them get $\bar{p}=\frac{1}{n}$ customers. If one firm deviates, it will be ranked first by half of the customers and last by the other half. So it will get $\frac{1}{2} p_{1}+\frac{1}{2} p_{n}$. We have a CNE iff $\frac{1}{2} p_{1}+\frac{1}{2} p_{n} \leq \bar{p}$. This is equivalent to $\frac{1}{2} p_{1}-\frac{1}{2} \bar{p} \leq \frac{1}{2} \bar{p}-\frac{1}{2} p_{n}$, which, in turn, is equivalent to $p_{1}-\bar{p} \leq \frac{1}{2}\left(p_{1}-p_{n}\right)$ or $c(\mathbf{p}, n) \leq \frac{1}{2}$.

The existence of CNE does not preclude the existence of NCNE-both can coexist for the same vector. For example, computations show that the vector $\mathbf{p}=$ $\frac{1}{14}(3,2,2,2,2,1,1,1,0)$ with $c(\mathbf{p}, 9)=\frac{3 / 14-1 / 9}{3 / 14}=\frac{13}{27}<\frac{1}{2}$ has CNE but also symmetric NCNE of type $(3,3,3)$.

\subsection{Initial investigation of market shares of firms}

Consider a strategy profile $x=\left(\left(x_{1}, n_{1}\right), \ldots,\left(x_{m}, n_{m}\right)\right)$ and a firm $f$ at location $x_{1}$, where there are $n_{1}$ firms in total. For any other occupied location $x_{j}, j \neq 1$, we draw 
Fig. 2 The market of location $x_{1}$ splits the circle into segments with equal probability of purchasing at location $x_{1}$

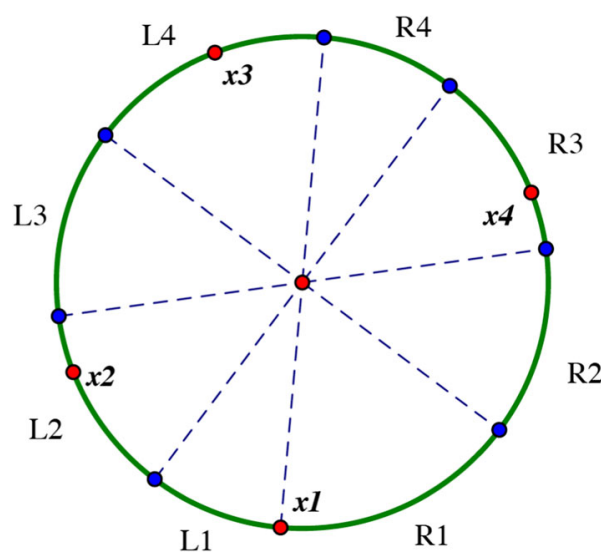

a diameter line $\ell_{j}$ that passes perpendicularly through the midpoint of the segment $x_{1} x_{j}$. This line intersects the circumference of the circle exactly at the two halfway points between the two locations $x_{1}$ and $x_{j}$, thus dividing the market into consumers who are closer to $x_{1}$ than $x_{j}$ and those who are closer to $x_{j}$ than $x_{1}$ (see Fig. 2).

In this way, we partition the market into regions of consumers who have the same ranking of firms at $x_{1}$, hence patronising them with equal probability. Also, draw a diameter line through $x_{1}$ and the center of the circle. This creates $2 m$ arc segments, where $m$ is the number of occupied locations - label them $L_{1}, \ldots, L_{m}$ in the clockwise direction from $x_{1}$ and $R_{1}, \ldots, R_{m}$ in the anticlockwise direction. We note that $\ell\left(L_{S}\right)=$ $\ell\left(R_{m-s+1}\right)$, where $\ell(A)$ is the length of $\operatorname{arc} A$.

For consumers in region $L_{s}$, all $n_{1}$ firms at location $x_{1}$, are tied for places from $k_{s}$ th to $\left(k_{s}+n_{1}-1\right)$ th, where $k_{1}=1$ and $k_{s}$ for $s \geq 2$ depends on the number of firms at other occupied positions. More precisely,

$$
k_{s}=1+n_{2}+\cdots+n_{s} .
$$

We also define $q_{1}=1$ and $q_{s}$ for $s \geq 2$ as

$$
q_{s}=1+n_{m}+n_{m-1}+\cdots+n_{m-s+2}
$$

Note that

$$
k_{s}+q_{m-s+1}=n-n_{1}+2 .
$$

We also note that for customers in $L_{1} \cup R_{1}$ firm $f$ will be tied for the first $n_{1}$ places and for customers in $L_{m} \cup R_{m}$ firm $f$ will be tied for the last $n_{1}$ places. From the region $L_{s}$ any firm at $x_{1}$ gets

$$
\left[\frac{1}{n_{1}} \sum_{j=k_{s}}^{k_{s}+n_{1}-1} p_{j}\right] \ell\left(L_{s}\right)
$$


consumers. Similarly, for consumers in region $R_{S}$, all firms at $x_{1}$ are tied for $q_{s}$ th, so that from this region any such firm gets

$$
\left[\frac{1}{n_{1}} \sum_{j=q_{s}}^{q_{s}+n_{1}-1} p_{j}\right] \ell\left(R_{S}\right)
$$

consumers. Thus, the overall consumer share of a firm $f$ at location $x_{1}$ is

$$
M(f)=\sum_{s=1}^{m}\left[\frac{1}{n_{1}} \sum_{j=k_{s}}^{k_{s}+n_{1}-1} p_{j}\right] \ell\left(L_{s}\right)+\sum_{s=1}^{m}\left[\frac{1}{n_{1}} \sum_{j=q_{s}}^{q_{s}+n_{1}-1} p_{j}\right] \ell\left(R_{s}\right) .
$$

Proposition 1 The market share of any location is a convex combination of the components of the vector of probabilities $\mathbf{p}$.

Proof It follows from (6) and the fact that $\sum_{s=1}^{m} \ell\left(L_{s}\right)+\sum_{s=1}^{m} \ell\left(R_{s}\right)=1$. So $M(f)$ is a convex combination of convex combinations of components of $\mathbf{p}$.

As in the case of plurality we can prove the following.

Lemma 1 The market share of location $x_{1}$ does not depend on the position of $x_{1}$ on the interior of the arc $x_{2} x_{m}$ to which $x_{1}$ belongs, all other things being equal. If all firms at location $x_{1}$ simultaneously change their location, the expected number of their customers remains constant unless they jump over some other firm or co-locate with the firms at $x_{2}$ or $x_{m}$.

Proof The median lines $\ell_{i}$ divide the market into consumers preferring location $x_{1}$ over $x_{i}$ and consumers preferring location $x_{i}$ over $x_{1}$. Together, all the median lines $\ell_{i}$, $i=2, \ldots, m$, partition the market into regions of consumers that all rank $x_{1}$ relative to other locations in the same way (see Fig. 2). If position $x_{1}$ moves slightly in one direction, say, counterclockwise by angle $\alpha$, then each of these median lines moves by half of this amount. That is, the angle between lines $\ell_{i}$ and $\ell_{j}$ is equal to the angle $\angle x_{i} x_{1} x_{j}$ that has its vertex at $x_{1}$ subtending the arc $x_{i} x_{j}$. When $x_{1}$ moves along the circle, this angle stays constant. Hence, lines $\ell_{j}, j=2, \ldots, r$ will be rotated by $\frac{1}{2} \alpha$. Since all customers in $L_{1} \cup R_{1}$ and $L_{m} \cup R_{m}$ rank firms at $x_{1}$ equivalently, the measure of consumers ranking firms at $x_{1}$ in a certain position is unchanged, as long as $x_{1}$ does not reach or go beyond $x_{2}$ or $x_{m}$.

We will also need the following technical lemma.

Lemma 2 Let's assume that in the profile $x=\left(\left(x_{1}, n_{1}\right), \ldots,\left(x_{m}, n_{m}\right)\right)$ we have $n_{1}>$ 1 and consider two potential infinitesimal deviations by one of the firms $f$ located at $x_{1}$ to the right and to the left. Let $M(f)$ be $f$ 's market share at $x_{1}$ and $M_{-}(f), M_{+}(f)$ be its market shares after clockwise and anticlockwise deviations, respectively. Then

$$
M_{-}(f)+M_{+}(f)-2 M(f)=\sum_{s=1}^{m}\left[p_{k_{s}}+p_{k_{s}+n_{1}-1}-\frac{2}{n} \sum_{j=k_{s}}^{k_{s}+n_{1}-1} p_{j}\right] \ell\left(L_{s}\right)
$$




$$
+\sum_{s=1}^{m}\left[p_{q_{s}}+p_{q_{s}+n_{1}-1}-\frac{2}{n_{1}} \sum_{j=q_{s}}^{q_{s}+n_{1}-1} p_{j}\right] \ell\left(R_{s}\right) .
$$

Proof An infinitesimal deviation clockwise gives a share of

$$
M_{-}(f)=\sum_{s=1}^{m} p_{k_{s}} \ell\left(L_{s}\right)+\sum_{s=1}^{m} p_{q_{s}+n_{1}-1} \ell\left(R_{s}\right),
$$

while a similar deviation counterclockwise would yield

$$
M_{+}(f)=\sum_{s=1}^{m} p_{k_{s}+n_{1}-1} \ell\left(L_{s}\right)+\sum_{s=1}^{m} p_{q_{s}} \ell\left(R_{s}\right) .
$$

Adding these two and subtracting (6) we get (7).

\section{Convex probability vectors}

We proceed to investigate the class of convex probability vectors, which capture a broad range of plausible descriptions of voter behavior. Cahan and Slinko (2017) showed that on a linear bounded space, under a convex probability vector, NCNE often do not exist, with a few notable exceptions such as certain truncated Borda vectors (which include plurality). This does not carry through to a circular space and we will show that every convex vector on a circular market has NCNE. Here is the formal definition of convexity.

Definition 3 A probability vector $\mathbf{p}=\left(p_{1}, \ldots, p_{n}\right)$ with $p_{1} \geq p_{2} \geq \ldots \geq p_{n}$ is convex if

$$
p_{1}-p_{2} \geq p_{2}-p_{3} \geq \ldots \geq p_{n-1}-p_{n} .
$$

We say $\mathbf{p}$ is strictly convex if all the inequalities are strict.

We note that as soon as $p_{i}=p_{i+1}$ for some $i$, all the subsequent probabilities must also be equal for convexity to be satisfied. Firstly, we note that a convex vector $\mathbf{p}$ is either best-rewarding or intermediate.

Proposition 2 Let $\mathbf{p}$ be a convex vector. Then $c(\mathbf{p}, n) \geq 1 / 2$.

Proof Suppose $\mathbf{p}$ satisfies (10). We have, for any $1 \leq i \leq\lfloor n / 2\rfloor$,

$$
p_{i}-p_{i+1} \geq p_{i+1}-p_{i+2} \geq \ldots \geq p_{n-i}-p_{n-i+1} .
$$

In particular, all we need is

$$
p_{i}-p_{i+1} \geq p_{n-i}-p_{n-i+1} .
$$


Suppose $n$ is even. Equation (11) implies

$$
p_{1}+p_{n} \geq p_{2}+p_{n-1} \geq \cdots \geq p_{n / 2}+p_{n / 2+1} .
$$

Then

$$
\begin{aligned}
\bar{p}=\frac{1}{n} \sum_{i=1}^{n} p_{i} & =\frac{\left(p_{1}+p_{n}\right)+\left(p_{2}+p_{n-1}\right)+\cdots+\left(p_{n / 2}+p_{n / 2+1}\right)}{n} \\
& \leq \frac{n / 2}{n}\left(p_{1}+p_{n}\right)=\frac{1}{2}\left(p_{1}+p_{n}\right)
\end{aligned}
$$

Suppose $n$ is odd. Then (11) implies

$$
p_{1}+p_{n} \geq p_{2}+p_{n-1} \geq \cdots \geq p_{(n-1) / 2}+p_{(n-1) / 2+2} \geq 2 p_{(n+1) / 2} .
$$

Then, letting $k=(n-1) / 2$ and using (13), we have

$$
\begin{aligned}
\bar{p}=\frac{1}{n} \sum_{i=1}^{n} p_{i} & =\frac{\left(p_{1}+p_{n}\right)+\cdots+\left(p_{k}+p_{k+2}\right)+p_{k+1}}{n} \\
& \leq \frac{k\left(p_{1}+p_{n}\right)+p_{k+1}}{n} \\
& \leq\left(\frac{1}{2}-\frac{1}{2 n}\right)\left(p_{1}+p_{n}\right)+\frac{1}{2 n}\left(p_{1}+p_{n}\right)=\frac{1}{2}\left(p_{1}+p_{n}\right) .
\end{aligned}
$$

So in both cases $p_{1}+p_{n} \geq 2 \bar{p}$, which is equivalent to $c(\mathbf{p}, n) \geq 1 / 2$.

Corollary $1 p_{i+1}+p_{i+n}-\frac{2}{n}\left(p_{i+1}+\cdots+p_{i+n}\right) \geq 0$.

Proof The proof is similar to the proof of Proposition 2.

Theorem 3 If $\mathbf{p}$ is convex, a NCNE of type $(1,1, \ldots, 1)$ exists where the firms are equally spaced.

Proof Consider a profile $x=\left(\left(x_{1}, 1\right), \ldots,\left(x_{n}, 1\right)\right)$, where $x_{1}, \ldots, x_{n}$ are the vertices of a regular $n$-gon inscribed into the unit circle. Suppose firm $f_{j}$ is located at $x_{j}$, $j=1, \ldots, n$. If one of the firms other than $f_{1}$, say $f_{j+1}$, where $j \geq 1$, deviates from position $x_{j+1}$, joining the firm $f_{1}$ at location $x_{1}$, then one diameter line $\ell_{j+1}$, corresponding to the segment $x_{1} x_{j+1}$, disappears. As a result, the $\operatorname{arcs} L_{j}$ and $L_{j+1}$ join, creating a new arc $L_{j}^{\prime}$ of length $\frac{1}{n}$.

Thus, the new profile will be $x^{\prime}=\left(\left(x_{1}, 2\right), \ldots,\left(x_{j}, 1\right),\left(x_{j+2}, 1\right),\left(x_{n}, 1\right)\right)$ with new $\operatorname{arcs} L_{1}^{\prime}, \ldots, L_{n-1}^{\prime}$ and $R_{1}^{\prime}, \ldots, R_{n-1}^{\prime}$ of lengths

$$
\begin{aligned}
& \ell\left(L_{s}^{\prime}\right)=\ell\left(L_{s}\right)=\frac{1}{2 n} \text { for } s=1, \ldots, j-1, \\
& \ell\left(L_{j}^{\prime}\right)=\frac{1}{n},
\end{aligned}
$$




$$
\ell\left(L_{s}^{\prime}\right)=\ell\left(L_{s+1}\right)=\frac{1}{2 n} \text { for } s=j+1, \ldots, n-1 .
$$

and

$$
\begin{aligned}
& \ell\left(R_{s}^{\prime}\right)=\ell\left(R_{s}\right)=\frac{1}{2 n} \text { for } s=1, \ldots, n-j, \\
& \ell\left(R_{n-j+1}^{\prime}\right)=\frac{1}{n}, \\
& \ell\left(R_{s}^{\prime}\right)=\ell\left(R_{s+1}\right)=\frac{1}{2 n} \text { for } s=n-j+2, \ldots, n-1 .
\end{aligned}
$$

Let $M^{\prime}\left(f_{i}\right)$ be the market share of firm $f_{i}$ after the deviation. Then $M^{\prime}\left(f_{j+1}\right)=M^{\prime}\left(f_{1}\right)$ and, by (6),

$$
\begin{aligned}
M^{\prime}\left(f_{j+1}\right)= & \frac{1}{2 n}\left[\sum_{s=1}^{j-2} \frac{p_{s}+p_{s+1}}{2}+2 \cdot \frac{p_{j}+p_{j+1}}{2}+\sum_{s=j+2}^{n-1} \frac{p_{s}+p_{s+1}}{2}\right] \\
& +\frac{1}{2 n}\left[\sum_{s=1}^{n-j-2} \frac{p_{s}+p_{s+1}}{2}+2 \cdot \frac{p_{n-j+1}+p_{n-j+2}}{2}+\sum_{s=n-j+3}^{n-1} \frac{p_{s}+p_{s+1}}{2}\right] \\
= & \frac{1}{2 n}\left[1-\frac{p_{1}+p_{n}}{2}+\frac{p_{j}+p_{j+1}}{2}\right] \\
& +\frac{1}{2 n}\left[1-\frac{p_{1}+p_{n}}{2}+\frac{p_{n-j+1}+p_{n-j+2}}{2}\right] .
\end{aligned}
$$

Subtracting this from $M\left(f_{j+1}\right)=\frac{1}{n}$ and using (12) and (13), we get

$$
M\left(f_{j+1}\right)-M^{\prime}\left(f_{j+1}\right)=\frac{1}{2 n}\left[p_{1}+p_{n}-\frac{p_{j}+p_{j+1}}{2}-\frac{p_{n-j+1}+p_{n-j+2}}{2}\right] \geq 0,
$$

and hence the move by firm $f_{j+1}$ is not profitable.

Let us now consider the case where a firm $f_{j+1}$ relocates to the interior of arc $x_{1} x_{2}$. Due to Lemma 1, we may assume that this firm locates on this arc $x_{1} x_{2}$ infinitesimally close to $x_{1}$. Then the new $\operatorname{arcs} L_{1}^{\prime}, \ldots, L_{n-1}^{\prime}$ and $R_{1}^{\prime}, \ldots, R_{n-1}^{\prime}$ will be as in the first case. Then the new market share $M^{\prime}\left(f_{j+1}\right)$ will be

$$
M^{\prime}\left(f_{j+1}\right)=\frac{1}{2 n}\left[\sum_{i=1}^{n-1} p_{i}+p_{j}\right]+\frac{1}{2 n}\left[\sum_{i=2}^{n} p_{i}+p_{n-j+1}\right]
$$

Then $M\left(f_{j+1}\right)-M^{\prime}\left(f_{j+1}\right)=p_{1}+p_{n}-p_{j}-p_{n-j+1} \geq 0$, which means that this deviation is also not profitable.

Let us now consider profiles where some firms are paired. 
Definition 4 We say that a strategy profile is a Local Nash Equilibrium (LNE) if no firm can get an advantage by moving to another location without crossing or joining any other firm, that is, strictly in the arc $x_{2} x_{m}$.

Theorem 4 If $\mathbf{p}$ is strictly convex, no local (and of course global) Nash equilibria exist with three or more firms at one of the locations.

Proof Suppose $\mathbf{p}$ is strictly convex. If $n_{1}>2$, then all the terms in square brackets in (7) of Lemma 2 are positive. As shown in Corollary 1, convexity of the probability vector implies $p_{(j-1) n_{1}+1}+p_{j n_{1}}-\frac{2}{n_{1}}\left(p_{(j-1) n_{1}+1}+\cdots+p_{j n_{1}}\right) \geq 0$ for any $j$ such that $j n_{1} \leq m$. This inequality is strict for strict convexity. So the inequality

$$
M_{-}(f)+M_{+}(f)-2 M(f) \leq 0
$$

cannot be satisfied.

For a convex (but not strictly convex) probability vector $\mathbf{p}$ to admit an equilibrium, it must be that every term in the brackets in (7) is equal to zero - that is, for each $s \in[m]$, the subvectors $\left(p_{k_{s}}, \ldots, p_{k_{s}+n-1}\right)$ and $\left(p_{q_{s}}, \ldots, p_{q_{s}+n-1}\right)$ must be linearly decreasing (or constant) in the index. In particular, the probability vector will initially be decreasing linearly in the index, after which at some point it may continue to decrease at a slower rate or stop decreasing and remain constant until the end. In such cases, equilibria can exist, as shown in the subsequent examples.

Example 1 Suppose there are two diametrically opposite locations on the circular market occupied by three firms-1,2 and 3-and two firms - 4 and 5-respectively. Let us calculate the probability vectors $\mathbf{p}$ for which this configuration is a NCNE. In this configuration, each firm among the triple gets

$$
\frac{1}{2} \cdot \frac{p_{1}+p_{2}+p_{3}}{3}+\frac{1}{2} \cdot \frac{p_{3}+p_{4}+p_{5}}{3}=\frac{1}{6}\left(p_{1}+p_{2}+2 p_{3}+p_{4}+p_{5}\right)
$$

customers. After a deviation by firm 1 anywhere except on top of firms 4 and 5 (in which case there is no change in market share), as shown in Fig. 3, firm 1 will get

$$
\frac{1}{4} p_{1}+\frac{1}{2} p_{3}+\frac{1}{4} p_{5}=\frac{1}{4}\left(p_{1}+2 p_{3}+p_{5}\right)
$$

For a NCNE, expression (16) must not exceed expression (15), which gives the inequality

$$
\frac{1}{4}\left(p_{1}+2 p_{3}+p_{5}\right) \leq \frac{1}{6}\left(p_{1}+p_{2}+2 p_{3}+p_{4}+p_{5}\right)
$$

or

$$
p_{1}-2 p_{2}+2 p_{3}-2 p_{4}+p_{5} \leq 0
$$


Fig. 3 The diagrams shows a deviation of firm 1

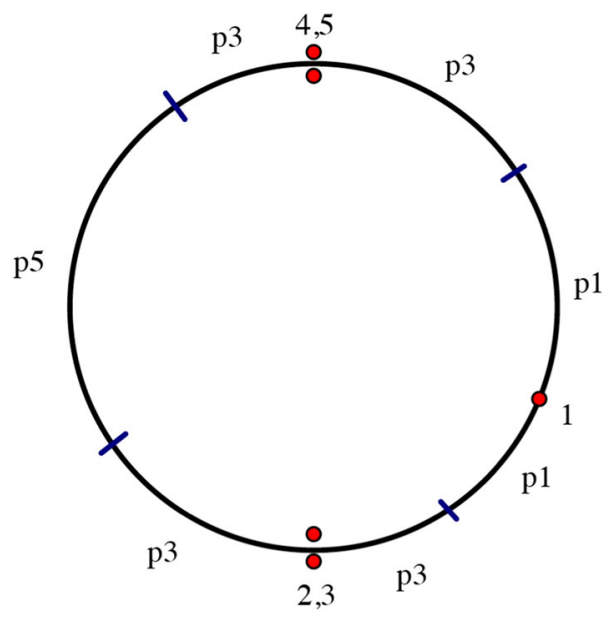

which can be rewritten as

$$
\left(p_{1}-2 p_{2}+p_{3}\right)+\left(p_{3}-2 p_{4}+p_{5}\right) \leq 0 .
$$

This is always satisfied for a concave $\mathbf{p}$, but never for a strictly convex vector. For a weakly convex vector, however, it can be satisfied. For example, consider $\mathbf{p}=$ $\frac{1}{13}(6,4,2,1,0)$. Also neither of the two firms at the other occupied position would get an advantage deviating to an unoccupied location. By Lemma 1 it is sufficient to consider only infinitesimal deviations. Deviating in any direction, the firm will not gain (or lose) anything, hence this deviation is not profitable.

For NCNE, it should also be the case that neither of these two paired firms wants to deviate to the other occupied position and join the three firms there. This yields the requirement that

$$
\frac{1}{2} \cdot \frac{p_{1}+p_{2}+p_{3}+p_{4}}{4}+\frac{1}{2} \cdot \frac{p_{2}+p_{3}+p_{4}+p_{5}}{4} \leq \frac{1}{2} \cdot \frac{p_{1}+p_{2}}{2}+\frac{1}{2} \cdot \frac{p_{4}+p_{5}}{2},
$$

which simplifies to $2 p_{3} \leq p_{1}+p_{5}$, which is true for a convex $\mathbf{p}$. Note that it is not true for a strictly concave vector, which, combined with (18), rules out NCNE for strictly concave vectors.

There are, however, convex vectors that satisfy both (18) and (19), and hence have NCNE! In particular, the vector

$$
\mathbf{p}=\frac{1}{13}(6,4,2,1,0)
$$

is of this kind. Apart from the equilibrium of type $(3,2)$, this vector also has equilibria of types $(1,1,1,1,1),(2,1,1,1),(2,2,1)$. 
Example 2 The vector

$$
\mathbf{p}=\frac{1}{21}(8,6,4,2,1,0)
$$

has NCNE of types $(1,1,1,1,1,1),(2,1,1,1,1),(2,2,1,1),(2,2,2),(3,3)$.

Now we will move towards a classification of types of NCNE for strictly convex vectors. Theorem 4 says that no more than two firms can co-locate in an equilibrium. The following lemma says that in equilibrium being paired is not an advantage.

Lemma 3 Let the vector $\mathbf{p}$ be strictly convex and a profile $x=\left(\left(x_{1}, n_{1}\right), \ldots,\left(x_{m}, n_{m}\right)\right)$ is a NE with $n_{1}=2$. Then for a firm at $x_{1}$ an infinitesimal deviation from $x_{1}$ does not change its market share.

Proof Since $n_{1}=2$, all square brackets in (7) vanish and the whole expression $M_{-}(f)+M_{+}(f)-2 M(f)$ for a firm $f$ at $x_{1}$ is always 0 . But in equilibrium $M_{-}(f) \leq M(f)$ and $M_{+}(f) \leq M(f)$. This implies that the firm's payoff upon either infinitesimal deviation is equal to the payoff if it does not deviate.

Due to Lemmas 1 and 3 it is tempting to suggest that if there is a NCNE, then there will be an equally spaced one. This, indeed, happens in many observed cases but not in all of them.

Example 3 The computational tool described in Sect. 9 shows for the vector $\mathbf{p}=$ $\frac{1}{42}(20,10,5,3,2,2)$ the existence of a Nash equilibria of type $(2,2,1,1)$, however, it can be easily manually checked that it cannot be equally spaced.

If we venture beyond convex vectors, in the class of best-rewarding vectors we can find some even more striking examples. In particular, we may note that the equilibria may be equally spaced but highly non-symmetric at the same time.

Example 4 The vector $\mathbf{p}=\frac{1}{12}(3,2,2,2,2,1,0,0,0)$ with $c(\mathbf{p}, 9)=\frac{3 / 12-1 / 9}{3 / 12}=\frac{5}{9}>$ $\frac{1}{2}$ has only Nash equilibria of types $(8,1)$ and $(7,1,1)$.

In particular, this example shows that, unlike convex vectors, for a best-rewarding vector we do not always have an equilibrium where all firms are non-paired and equally spaced. This justifies emphasizing the class of convex vectors within the class of best-rewarding vectors.

\section{Intermediate probability vectors}

Computations indicate that some intermediate vectors may have NCNE of all possible types. In particular, this is true for the Borda vector and $n$-dimensional vectors

$$
\mathbf{p}=\frac{1}{n}(2,1, \ldots, 1,0)
$$

However, for the vector $\mathbf{p}=\frac{1}{20}(7,6,3,3,1)$ we have only equilibria of type $(3,2)$. The difference between the two cases is that in the first case the vectors are symmetric while the latter vector is not. 
Definition 5 A vector $\mathbf{p}$ is said to be symmetric if for all $s$,

$$
\frac{p_{s}+p_{n-s+1}}{2}=\bar{p}
$$

A symmetric vector rewards increases in ranking at top positions the exact same way it punishes decreases in ranking at bottom positions. It is easy to see that any symmetric vector is intermediate.

Theorem 5 If $\mathbf{p}$ is symmetric, any strategy profile is a Nash equilibrium. In particular, all firms get an equal share of the market.

Proof Let $\mathbf{p}$ be an intermediate vector and $x=\left(\left(x_{1}, n_{1}\right), \ldots,\left(x_{m}, n_{m}\right)\right)$ be a profile. Let us calculate the share of a firm $f$ at location $x_{1}$. For some $s$, such that $1 \leq s \leq m$, consider the contribution of customers from regions $L_{s}$ and $R_{m-s+1}$ which lie opposite to each other (see Fig. 2). Due to (4) and (5) the customers from $L_{s}$ and $R_{m-s+1}$ contribute

$$
M_{s}(f)=\frac{1}{n_{1}}\left[\sum_{j=k_{s}}^{k_{s}+n_{1}-1} p_{j}+\sum_{j=q_{m-s+1}}^{q_{m-s+1}+n_{1}-1} p_{j}\right] A_{s}
$$

where $A_{s}=\ell\left(L_{s}\right)=\ell\left(R_{m-s+1}\right)$. We note now that by (3)

$$
\left(k_{s}+i\right)+\left(q_{m-s+1}+n_{1}-i-1\right)=n+1
$$

for all $i=0,1, \ldots, n_{1}-1$. This means by (20) that

$$
p_{k_{s}+i}+p_{q_{m-s+1}+n_{1}-i-1}=2 \bar{p}
$$

so the aforementioned contribution is $2 \bar{p} A_{s}$. Overall, the market share of firm $f$ is $M(f)=\sum_{s=1}^{m} 2 \bar{p} A_{s}=2 \bar{p} \frac{1}{2}=\bar{p}$. This proves the theorem.

On the other hand, some intermediate vectors have just a few equilibria. Some of them, like $\mathbf{p}=\frac{1}{24}(7,6,4,3,3,1)$, have only two types of clustered equilibria, namely, of types $(2,2,2)$ and $(3,3)$.

In general, we know very little about non-symmetric intermediate vectors. In particular, we do not know if all of them have NCNE. Computations show that this may be the case.

\section{Concave probability vectors}

Next, we look at concave probability vectors. This class of vectors represents the opposite side of the coin to convex vectors, and may be a plausible model of consumer behavior in some settings. 
Definition 6 A probability vector $\mathbf{p}=\left(p_{1}, \ldots, p_{n}\right)$ with $p_{1} \geq p_{2} \geq \ldots \geq p_{n}$ is concave if

$$
p_{1}-p_{2} \leq p_{2}-p_{3} \leq \ldots \leq p_{n-1}-p_{n} .
$$

We say $\mathbf{p}$ is strictly concave if all the inequalities are strict.

Firstly, we note that a concave vector is worst-punishing.

Proposition 3 Let $\mathbf{p}$ be a concave vector. Then $c(\mathbf{p}, n) \leq 1 / 2$.

Proof Same argument as in Proposition 2, just reverse the inequality signs.

Since they are worst-punishing, concave vectors produce incentives for central agglomeration, allowing CNE over the central segment of the market in the linear case (Cahan and Slinko 2017). NCNE are not known to exist (but nonexistence is also unproved). On the circular market, the absence of the "peripheral firm advantage" may possibly allow for a much broader range of equilibria exhibiting the realistic property of multilocational clustering of multiple firms. Yet, like in the linear case, we have not found any so far. Intuitively, if a NCNE existed, it is likely to be a bipositional one. Corollary 2, however, rules out such NCNE entirely. This supports the conjecture that, for strictly concave vectors, NCNE do not exist even on a circular market without boundary conditions.

Next we show that half or more of the firms involved cannot co-locate. Exactly as in the convex case (12) we can obtain

$$
p_{1}+p_{n} \leq p_{2}+p_{n-1} \leq \cdots \leq p_{n / 2}+p_{n / 2+1}
$$

if $n$ is even, and

$$
p_{1}+p_{n} \leq p_{2}+p_{n-1} \leq \cdots \leq p_{(n-1) / 2}+p_{(n-1) / 2+2} \leq 2 p_{(n+1) / 2}
$$

if $n$ is odd. For strict concavity all the inequalities turn out to be strict.

Suppose $\mathbf{p}$ is strictly concave. For convenience, we assume that the number of firms is even so that $n=2 k$ where $k$ is a positive integer. The case where $n$ is odd is similar, with the use of (24) instead of (23). Firstly, we note that for any $2 \leq t \leq k$

$$
\frac{1}{n}=\bar{p}=\frac{\left(p_{1}+p_{n}\right)+\left(p_{2}+p_{n-1}\right)+\cdots+\left(p_{k}+p_{k+1}\right)}{n}<\frac{1}{n-2 t+2} \sum_{i=t}^{n-t+1} p_{i} .
$$

Lemma 4 Let $\mathbf{p}$ be strictly concave, $n=2 k$, and $x=\left(\left(x_{1}, n_{1}\right), \ldots,\left(x_{m}, n_{m}\right)\right)$ be a profile where $m \geq 2$ and $n_{1} \geq k$. Then the market share $M(f)$ of a firm $f$ at $x_{1}$ is greater than or equal to $\bar{p}=\frac{1}{n}$. Moreover, $M(f)>\frac{1}{n}$, if $m>2$ or $n_{1}>k$. 
Proof The number of customers received by a firm $f$ in the large cluster is given by (6). However, we know that $\ell\left(L_{s}\right)=\ell\left(R_{m-s+1}\right)$ and, by (3), $q_{m-s+1}=n-n_{1}-k_{s}+2$. Taking this to account we get

$$
M(f)=\sum_{s=1}^{m} \frac{1}{n_{1}}\left[\sum_{j=k_{s}}^{k_{s}+n_{1}-1} p_{j}+\sum_{j=n-n_{1}-k_{s}+2}^{n-k_{s}+1} p_{j}\right] A_{s}=\sum_{s=1}^{m} M_{s}(f),
$$

where $M_{s}(f)$ is given in (21). We note that if $n_{1}=k$, then $n_{1}$ and $n-n_{1}+1=k+1$ are two consecutive integers and it is easy to see that in such a case

$$
M_{1}(f)=M_{m}(f)=\frac{2}{n} A_{1} .
$$

If $n_{1}>k$, then we have $n_{1}>n-n_{1}+1$. Because of that, if $s=1$, due to (25), the corresponding term in the sum (26) will be

$$
\begin{aligned}
M_{1}(f) & =\frac{1}{n_{1}}\left[\sum_{j=1}^{n_{1}} p_{j}+\sum_{j=n-n_{1}+1}^{n} p_{j}\right] A_{1}=\frac{1}{n_{1}}\left[1+\sum_{j=n-n_{1}+1}^{n_{1}} p_{j}\right] A_{1} \\
& >\frac{1}{n_{1}}\left[1+\frac{2 n_{1}-n}{n}\right] A_{1}=\frac{2}{n} A_{1} .
\end{aligned}
$$

Similarly,

$$
M_{m}(f)>\frac{2}{n} A_{m}
$$

If there are at least three locations, i.e., $m \geq 3$, then for any positive integer $s$ such that $1<s<m$, due to (25), we would have

$$
\begin{aligned}
M_{s}(f) & =\frac{1}{n_{1}}\left[\sum_{j=k_{s}}^{k_{s}+n_{1}-1} p_{j}+\sum_{j=n-n_{1}-k_{s}+2}^{n-k_{s}+1} p_{j}\right] A_{s} \\
& =\frac{1}{n_{1}}\left[\sum_{j=k_{s}}^{n-k_{s}+1} p_{j}+\sum_{j=n-n_{1}-k_{s}+2}^{k_{s}+n_{1}-1} p_{j}\right] A_{s} \\
& >\frac{1}{n_{1}}\left[\frac{n-2 k_{s}+2}{n}+\frac{2 n_{1}+2 k_{s}-n-2}{n}\right] \ell\left(L_{s}\right)=\frac{2}{n} A_{s} .
\end{aligned}
$$

Overall, if $m>2$ or $n_{1}>k$, then

$$
M(f)>\frac{2}{n} \sum_{i=1}^{m} A_{i}=\frac{2}{n} \cdot \frac{1}{2}=\frac{1}{n} .
$$

Alternatively, if $m=2$ and $n_{1}=k$, we have $M(f)=\frac{1}{n}$. 
Theorem 6 For any strictly concave vector, no NCNE exist with a location occupied by half or more of the firms.

Proof Let $m \geq 2$ and $n_{1} \geq k$. If $m>2$ or $n_{1}>k$, then any firm at $x_{1}$ will have a greater than average market share. Then some firm located at one of the other locations will have a market share smaller than average. If it moves to join a cluster at $x_{1}$, then by Lemma 4 in the new position it will have a market share greater than average, hence the move will be profitable. If $m=2$ and $x_{1}=k$, then there will be two clusters of equal sizes at $x_{1}$ and $x_{2}$. Every firm will have an average market share. But if any of the firms joins the other cluster, by Lemma 4 it will do better than average. Hence the move is profitable.

Corollary 2 For any strictly concave vector there is no bipositional NCNE.

Proof Follows from Theorem 6 since, if we have only two positions, one of them will have at least half of all firms.

For concave vectors, this shows that critical mass, at which point all firms will eventually want to converge to a singularity, occurs when the majority of the firms are at the same location.

We conjecture that if $\mathbf{p}$ is strictly concave, no NCNE exist.

\section{The case of four firms}

For $n=4$, the types of equilibria that exist can be fully described. Firstly, we note that the condition $c(\mathbf{p}, 4) \geq 1 / 2$ is equivalent to

$$
p_{1}-p_{2}-p_{3}+p_{4} \geq 0 \text {. }
$$

Indeed, $c(\mathbf{p}, 4) \geq 1 / 2$ can be rewritten as $2\left(p_{1}-\bar{p}\right) \geq p_{1}-p_{4}$, which is equivalent to (27). Similarly, $c(\mathbf{p}, 4) \leq 1 / 2$ will be equivalent to

$$
p_{1}-p_{2}-p_{3}+p_{4} \leq 0 \text {. }
$$

We note that condition (27) is weaker than convexity. For example, $\mathbf{p}=\frac{1}{15}(7,5,2,1)$ is not convex but satisfies (27). Similarly, (28) is weaker than concavity. Hence, we are not able to use the results of Sects. 4 and 6.

Theorem 7 Let $n=4$ and $\mathbf{p}$ be a probability vector. Then:

(i) For all best-rewarding (but not intermediate) vectors, NCNE exist of types

$$
(1,1,1,1),(2,1,1),(2,2) \text {; }
$$

(ii) For all worst-punishing (but not intermediate) vectors, only CNE exist;

(iii) For an intermediate vector, all types of equilibria exist. 
Proof To show the existence of an equilibrium of type $(1,1,1,1),(2,1,1),(2,2)$, or $(3,1)$, it is enough to show there is an equally spaced one. The proof that a NCNE of type $(1,1,1,1)$ exists is very similar to the proof of Theorem 3. Similarly for $(2,1,1)$. Let us consider the configuration of type $(2,2)$.

Suppose there are two paired firms at diametrically opposite locations on a circular market. Then, for any probability vector $\mathbf{p}$, it is not profitable for any firm to deviate to any unoccupied location. Indeed, in the described configuration, due to symmetry, each firm gets $\frac{1}{4}$ of the customers. This is exactly the same as what firm 1 gets after a deviation to anywhere other than on top of firms 3 and 4 . This deviation is shown in Fig. 4. However, joining firms 3 and 4 will be profitable for any worst-punishing but not intermediate vector. In such a case its new share of customers will be

$$
s=\frac{1}{2}\left(\frac{p_{1}+p_{2}+p_{3}}{3}+\frac{p_{2}+p_{3}+p_{4}}{3}\right)=\frac{1}{6}\left(p_{1}+2 p_{2}+2 p_{3}+p_{4}\right) .
$$

Thus, in order for NCNE to exist, we must have

$$
\frac{1}{4}-s=\frac{1}{12}\left(-p_{1}+p_{2}+p_{3}-p_{4}\right) \geq 0
$$

This is the case for best-rewarding or intermediate $\mathbf{p}$ due to (27). Hence, the case of two oppositely located pairs of firms is a NCNE only for best-rewarding or intermediate vectors.

The same argument shows that, for a best-rewarding vector, the configuration with three firms at one location and one at another is not a NE since one firm from the location with three firms would be better off moving to the other location. This configuration is not a NCNE for worst-punishing vectors either since the lone firm would be better off joining the three other firms. The only case when a NCNE of type $(3,1)$ exists is when the vector is intermediate, in which case we have equality in (27).

\section{Computational tool}

To find NCNE for a particular vector of probabilities, we developed the software "round voter", which is based upon the "move voter" software created earlier. Both are available at: https://github.com/gmatht/MoveVoters.

The algorithm first considers each possible clustering of firms. For example, with three firms it considers the configurations $(1,1,1),(2,1)$, and $(1,2)$. It does not consider (3) as this represents a convergent solution, which is straightforward to deal with by Theorem 2. For each of the clusterings it creates a Linear Programme (LP). Given a clustering, for each separate location at which firms cluster, the LP has a position variable representing this location. We want these variables to be distinct, yet LPs do not allow strict inequalities. For this reason we also have a slack variable used to enforce a minimum distance between the positions. We maximize the slack variable and, if the maximum slack is zero, we reject the solution of the LP. As we maximize the slack variable we also find an equally spaced solution, if one exists. 

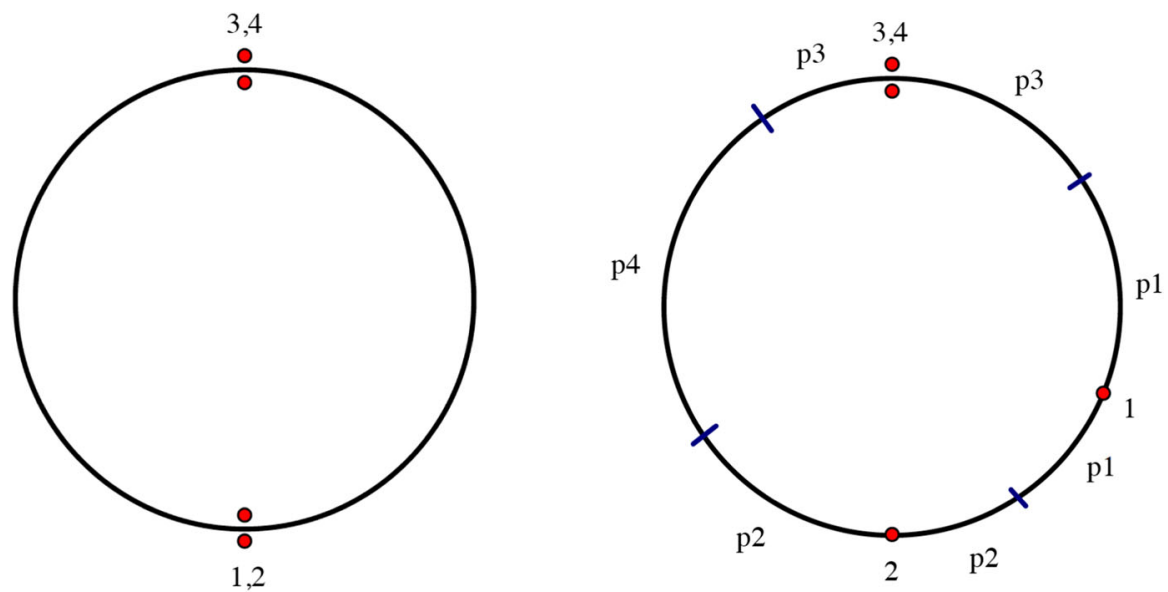

Fig. 4 The right diagrams shows a deviation of firm 1 from the location where it was paired with firm 2. The firm 1 gets the same, firm 2 gains and firms 3 and 4 lose

The LP has $3 m^{2}$ rows to ensure that the firms cannot gain an advantage by changing position, where $m$ is the number of distinct locations where firms cluster. For each position variable $i$ and position variable $j$, we include 3 rows representing the advantage a firm at position $i$ could get by moving to: just before position $j$, exactly position $j$, or just after position $j$. We require that all of these be at most zero.

The LP also has rows to ensure the variables represent the type of solution we are looking for. One row ensures that the first position variable is at least 0 ; a number of rows ensure each position is at least slack more than the position preceding it; another row ensures the final position is at least slack less than 1.

\section{Conclusion}

In many settings, it makes sense to model the market on which firms compete as a circle a là Salop (1979). At the same time, it is often the case that consumers shop at firms other than the nearest with some probability. In this paper we make substantial progress in investigating the nature of Nash equilibria, in particular, non-convergent Nash equilibria. We study several broad classes of probability vectors that realistically represent different kinds of consumer behavior. For convex probability vectors and symmetric probability vectors, non-convergent Nash equilibria are quite abundant. For concave probability vectors, our results point to the possibility that they do not exist at all. There are parallels to the classical case of a linear unit interval market (Cahan and Slinko 2017), but important differences are due to the different nature of the markets studied, particularly the lack of boundary conditions in the circular market case. 
Acknowledgements We thank referees for the careful reading of the manuscript and valuable remarks. Arkadii Slinko was supported by the Faculty Development Research Fund 3719899 of the University of Auckland.

Funding Open Access funding enabled and organized by CAUL and its Member Institutions

Open Access This article is licensed under a Creative Commons Attribution 4.0 International License, which permits use, sharing, adaptation, distribution and reproduction in any medium or format, as long as you give appropriate credit to the original author(s) and the source, provide a link to the Creative Commons licence, and indicate if changes were made. The images or other third party material in this article are included in the article's Creative Commons licence, unless indicated otherwise in a credit line to the material. If material is not included in the article's Creative Commons licence and your intended use is not permitted by statutory regulation or exceeds the permitted use, you will need to obtain permission directly from the copyright holder. To view a copy of this licence, visit http://creativecommons.org/licenses/by/4.0/.

\section{References}

Aoyagi M, Okabe A (1993) Spatial competition of firms in a two-dimensional bounded market. Reg Sci Urban Econ 23(2):259-289

Cahan D, McCabe-Dansted J, Slinko A (2018) Asymmetric equilibria in spatial competition under weakly concave scoring rules. Econ Lett 167:71-74

Cahan D, Slinko A (2017) Nonconvergent electoral equilibria under scoring rules: beyond plurality. J Public Econ Theory 19:445-460

Cahan D, Slinko A (2018) Electoral competition under best-worst voting rules. Soc Choice Welf 51:259-279

Coughlin PJ (1992) Probabilistic voting theory. Cambridge University Press, New York

Cox GW (1987) Electoral equilibrium under alternative voting institutions. Am J Polit Sci 31:82-108

Denzau A, Kats A, Slutsky S (1985) Multi-agent equilibria with market share and ranking objectives. Soc Choice Welf 2:96-117

De Palma A, Hong G, Thisse JF (1990) Equilibria in multi-party competition under uncertainty. Soc Choice Welf 7:247-259

Downs A (1957) An economic theory of political action in a democracy. J Polit Econ 65:135-150

Duggan J (2005) A survey of equilibrium analysis in spatial models of elections. Unpublished manuscript available at http://www.rochester.edu/College/PSC/duggan/papers/existsurvey4.pdf

Eaton CB, Lipsey RG (1975) The principle of minimum differentiation reconsidered: some new developments in the theory of spatial competition. Rev Econ Stud 42:27-49

Graitson D (1982) Spatial competition a la Hotelling: a selective survey. J Ind Econ 31:11-25

Hotelling H (1929) Stability in competition. Econ J 39:41-59

Myerson RB (1999) Theoretical comparisons of electoral systems. Eur Econ Rev 43:671-697

Peeters RJ, Saran R, Yüksel AM (2010) Strategic party formation on a circle. METEOR: Maastricht Research School of Economics of Technology and Organizations

Peeters RJ, Saran R, Yüksel AM (2016) Strategic party formation on a circle and Duverger's law. Soc Choice Welf 47(3):729-759

Salop SC (1979) Monopolistic competition with outside goods. Bell J Econ 10(1):141-156

Publisher's Note Springer Nature remains neutral with regard to jurisdictional claims in published maps and institutional affiliations. 